\title{
The Origin of UV-optical Variability in AGN and Test of Disc Models: XMM-Newton and ground based observations of NGC4395
}

\author{
I.M. $M^{c}$Hardy $^{1, \star}$ S. D. Connolly ${ }^{1}$, B. M. Peterson ${ }^{2}$, A. Bieryla ${ }^{3}$, H. Chand ${ }^{4}$, M.S. Elvis ${ }^{3}$, D. \\ Emmanoulopoulos $^{1}$, E. Falco ${ }^{3}$, P. Gandhi ${ }^{1}$, S. Kaspi ${ }^{5}$, D. Latham ${ }^{3}$, P. Lira ${ }^{6}$, C. McCully ${ }^{7}$, H. Netzer \\ and M. Uemura ${ }^{8}$ \\ 1 Department of Phyiscs and Astronomy, University of Southampton, University Road, Southampton SO17 1BJ, UK. \\ 2 LCOGT and Department of Astronomy, Ohio State University, 140 West 18th Avenue, Columbus, OH 43210-1173 \\ 3 Harvard Smithsonian Center for Astrophysics, 60 Garden Street, Cambridge, MA02138, USA. \\ 4 Aryabhatta Research Institute of Observational Sciences (ARIES), Manora Peak, Nainital 263 002, India \\ 5 Wise Observatory and School of Physics and Astronomy, Tel Aviv University, Tel Aviv 69978, Israel \\ 6 Departmento de Astronomia, Universidad de Chile, Camino del Observatorio 1515, Santiago, Chile \\ 7 LCOGT and Department of Physics and Astronomy, Rutgers University, 136 Frelinghuysen Road, Piscataway, NJ 0885, \\ USA \\ ${ }^{8}$ Hiroshima Astrophysical Science Center, Hiroshima University, Kagamiyama 1-3-1, Hiroshima 739-8526, Japan
}

Received October 2015, accepted November 2015

Published online later

Key words AGN; X-rays; UV; Optical; Variability; Accretion discs

The origin of short timescale (weeks/months) variability of AGN, whether due to intrinsic disc variations or reprocessing of X-ray emission by a surrounding accretion disc, has been a puzzle for many years. However recently a number of observational programmes, particularly of NGC5548 with Swift, have shown that the UV/optical variations lag behind the $\mathrm{X}$-ray variations in a manner strongly supportive of X-ray reprocessing. Somewhat surprisingly the implied size of the accretion disc is $\sim 3 \times$ greater than expected from a standard, smooth, Shakura-Sunyaev thin disc model. Although the difference may be explained by a clumpy accretion disc, it is not clear whether the difference will occur in all AGN or whether it may change as, eg, a function of black hole mass, accretion rate or disc temperature. Measurements of interband lags for most AGN require long timescale monitoring, which is hard to arrange. However for low mass $\left(<10^{6} M_{\odot}\right) \mathrm{AGN}$, the combination of XMM-Newton EPIC (X-rays) with the optical monitor in fast readout mode allows an X-ray/UVoptical lag to be measured within a single long observation. Here we summarise previous related observations and report on XMM-Newton observations of NGC4395 (mass 100× lower, accretion rate $\sim 20 \times$ lower than for NGC5548). We find that the UVW1 lags the X-rays by $\sim 470$ s. Simultaneous observations at 6 different ground based observatories also allowed the g-band lag $(\sim 800 s)$ to be measured. These observations are in agreement with X-ray reprocessing but initial analysis suggests that, for NGC4395, they do not differ markedly from the predictions of the standard thin disc model.

(c) 2016 WILEY-VCH Verlag GmbH \& Co. KGaA, Weinheim

\section{Models for UV/Optical Variability and relationship to X-ray Variability}

The origin of UV/optical variability in AGN and its relationship to X-ray variability has been a puzzle for some time and there are two main possibilities for the origin of the UV/optical variability. The UV/optical variability could result from reprocessing of $\mathrm{X}$-ray emission by the accretion disc or it could simply be the result of intrinsic variability of the thermal emission from the disc. These two models can, in principle, be distinguished simply by measuring the lag between the X-ray and UV/optical wavebands. In the reprocessing model, the UV/optical variations will lag behind the X-ray variations by the light travel time between the two emission regions. For a typical AGN this time will be a few hours. If the UV/optical variations are produced by intrinsic

\footnotetext{
* Corresponding author: e-mail: imh@ soton.ac.uk
}

disc variations there are two possible lag timescales. If the UV/optical photons are the seed photons for the X-ray emission, being Compton up-scattered in the central corona, then if the $\mathrm{X}$-ray variations are driven by seed photon variations, the X-ray emission will lag behind the UV/optical variations by the light travel time between the two emission regions, ie a few hours. Alternatively, if the UV/optical variations are caused by inwardly propagating accretion rate variations (Arévalo \& Uttley 2006), these variations will eventually propagate in at the viscous timescale and will affect the $\mathrm{X}$-ray emission region. In this case the $\mathrm{X}$-ray variations will lag the UV/optical varations by $\sim$ years.

\section{Previous Related Observations}

\subsection{UV/Optical inter-band lags}

The thin disc model which has been our accepted model for the temperature structure of optically thick accretion 
discs for over 40 years (Shakura \& Sunyaev 1973, SS) predicts a disc temperature profile $T \propto R^{-3 / 4}$. Therefore, in the $\mathrm{X}$-ray reprocessing model, in which incident $\mathrm{X}$-ray emission boosts the existing thermal emission from the disc, we expect that the time lag of the UV/optical variations after the X-ray variations should be given by lag $\propto$ wavelength $(\lambda)^{4 / 3}$. Sergeev et al. (2005) and Cackett et al. (2007) have measured the lags of the V, R, R1 and I bands relative to the B band for a sample of AGN. Cackett et al. find that, although not a perfect fit, the lags are broadly consistent with the prediction of a reprocessing model. However there were no accompanying X-ray measurements so it is unknown as to how the $\mathrm{X}$-ray variations might be related to the optical variations.

\subsection{RXTE and ground based optical observations}

To investigate the link between the X-ray emission and the UV/optical emission in AGN a number of groups (e.g. Arévalo et al. 2008, 2009; Breedt 2010; Breedt et al. 2009, 2010; Lira et al. 2011, 2015; Suganuma et al. 2006; Uttley et al. 2003) have monitored AGN quasisimultaneously in X-rays with RXTE and in optical wavebands from the ground (eg Fig. 11).

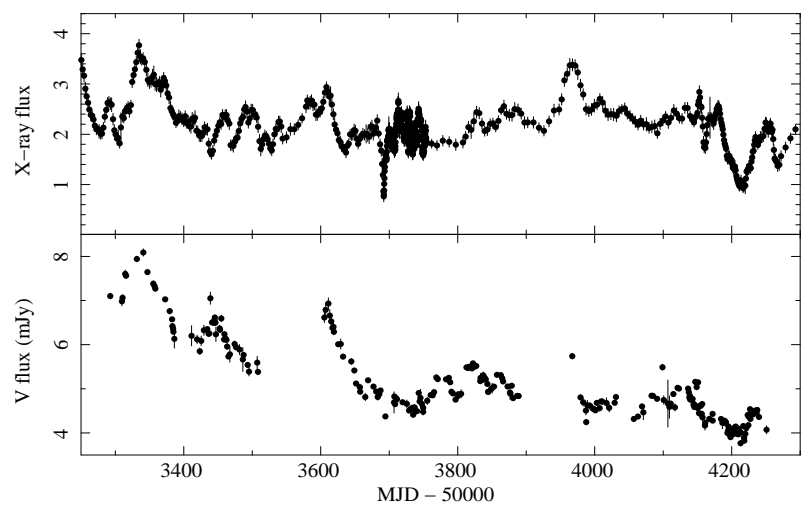

Fig. 1: RXTE X-ray (upper panel) and ground based Vband (lower panel) lightcurves of Mkn79 from Breedt et al. (2009).

Cross-correlation analysis, in all cases, shows either that the optical lags the X-rays by $\sim 1$ d (e.g. Fig. 2), or that there is no measurable lag. However the average sampling was $\sim 2 \mathrm{~d}$ and so the lags were rarely measured to better than $0.5 \mathrm{~d}$ and it was not possible to be absolutely certain that the $\mathrm{X}$-rays never led the optical.

\subsection{XMM-Newton and Swift single band lags}

To refine the measurement of the X-ray/optical lag better sampling is needed than was available with the RXTE and ground based optical monitoring. Such sampling is possible with Swift and with XMM-Newton. Swift allows observa-

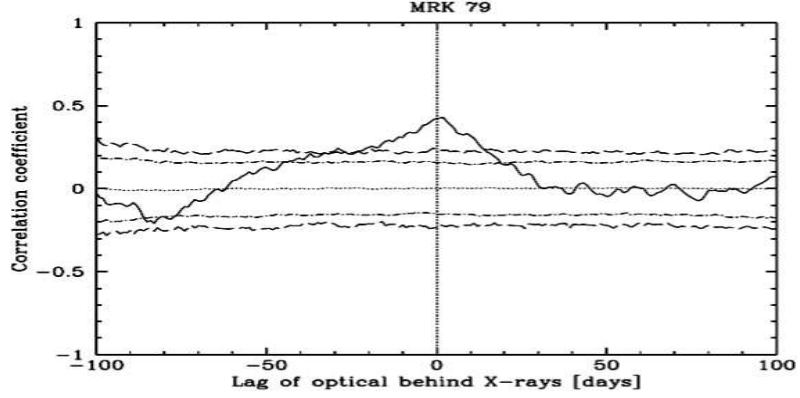

Fig. 2: Interpolation cross-correlation function (White \& Peterson 1994) between the X-ray and V-band lightcurves shown in Fig. 11 The V-band lags the X-rays by $\sim 1$ d (from Breedt et al. 2009) Here, and throughout this paper, a positive lag means that the longer wavelength lags behind the shorter wavelength.

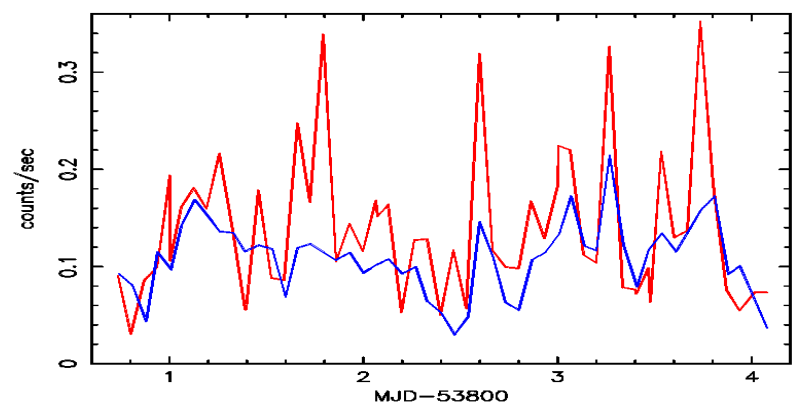

Fig. 3: Swift X-ray and B-band lightcurves of NGC4395 (from Cameron et al. 2012).

tions in the 0.5-10 keV X-ray band with the X-Ray Telescope (XRT) and, with the UV-Optical Telescope (UVOT), simultaneous observations in one of either 3 UV (UVW2, UVM2 and UVW1) or 3 optical (U, B and V) bands, depending on filter selection. XMM-Newton allows X-ray observations with EPIC in a similar band to Swift, and also allows UV/optical observations with the Optical Monitor (OM), which is similar to the Swift UVOT.

In Fig. 3 we show Swift X-ray and B-band variations in the low black hole mass AGN NGC4395 (mass $3.6 \times 10^{5} M_{\odot}$ - see Bentz and Katz http://www.astro.gsu.edu/AGNmass/ for all masses and luminosities). The data here are presented with a resolution of one satellite orbit (96 minutes). We can see a strong correlation and cross-correlation analysis (not shown here) reveals that the B-band lags the X-rays by less than the orbital sampling time (Cameron et al. 2012).

To obtain still higher time resolution it is possible to split the Swift orbital observations, of total duration $\sim 1000$ 1500s, into smaller time bins, eg 100 or 200s. In Fig. 4 we show the DCF derived from Swift X-ray and UVW2band observations with 200s time resolution (lightcurves not shown here). Although with large errors, this DCF suggests that the UVW2-band lags the X-rays by $\sim 400$ s. This lag is approximately what we expect based on X-ray repro- 


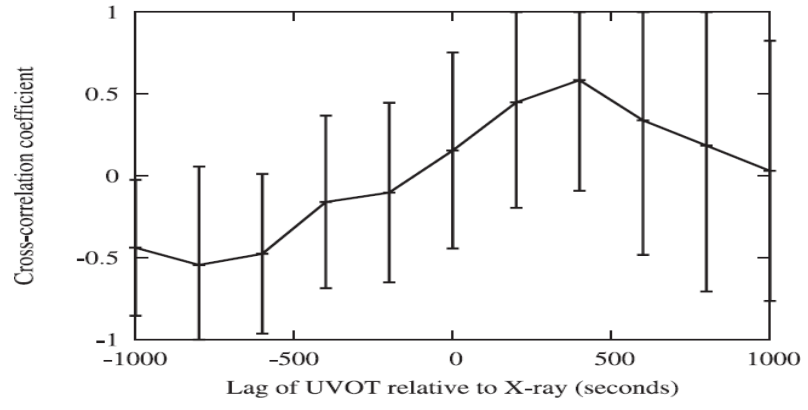

Fig. 4: Discrete correlation function (DCF Edelson \& Krolik 1988) derived from Swift eventmode UVOT data and X-ray photon counting data. The UVW2 lags behind the X-rays by $\sim 400$ s, although at low significance (from Cameron et al. 2012)

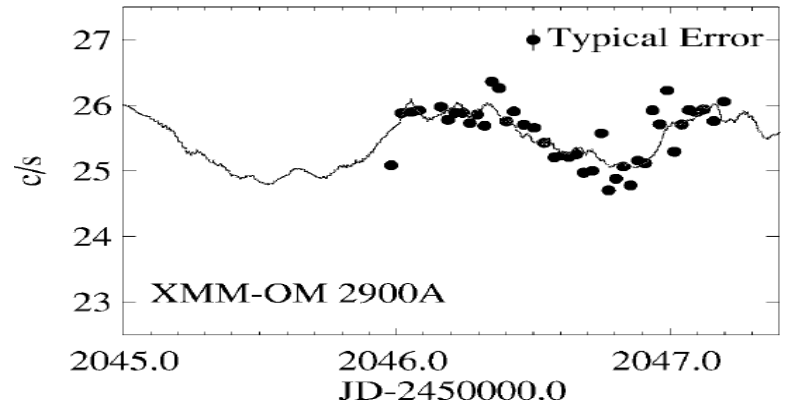

Fig. 5: XMM-Newton OM UVW1 imaging observations (black dots) of NGC4051 with 1ks exposure superposed on a model X-ray lightcurve from EPIC and RXTE observations, reprocessed by a ring of radius $0.14 \mathrm{~d}$ (from Mason et al. 2002).

cessing and formed the basis of the exciting XMM-Newton observations which we describe in Section 3

With XMM-Newton the OM has, until our observations which we report here in Section 3, usually been used in imaging mode. This mode provides a minimum exposure time of 800 s with a typical 300s readout time, thus limiting time resolution to about $1100 \mathrm{~s}$. Observations in this mode have not, in general, found significant correlations between the X-ray and UV/optical bands (Smith \& Vaughan 2007). One exception is NGC4051 (Mason et al. 2002) where, at $85 \%$ confidence, the UVW1 band was seen to lag the X-rays by $\sim 0.2 \mathrm{~d}$ (Fig. 5), broadly consistent with reprocessing.

\subsection{Swift multi-band lags}

A number of programmes have been undertaken with Swift to measure the lags between the X-ray band and the 6 UVOT bands. Shappee et al. (2014) presented lag measurements of the Seyfert galaxy NGC2617 but the results are slightly puzzling. The lags increase with wavelength, but if the fit is forced to go through the X-ray point then, for $\operatorname{lag} \propto \lambda^{\beta}$, they find $\beta=0.37$, which is far from the $4 / 3$ expected from reprocessing. If the X-ray point is ignored, a fit of $\beta=1.18$

is found between the other bands, but if extrapolated to the $\mathrm{X}$-ray wavelength, the fit is offset from the $\mathrm{X}$-ray point by a very large lag of $2.4 \mathrm{~d}$.

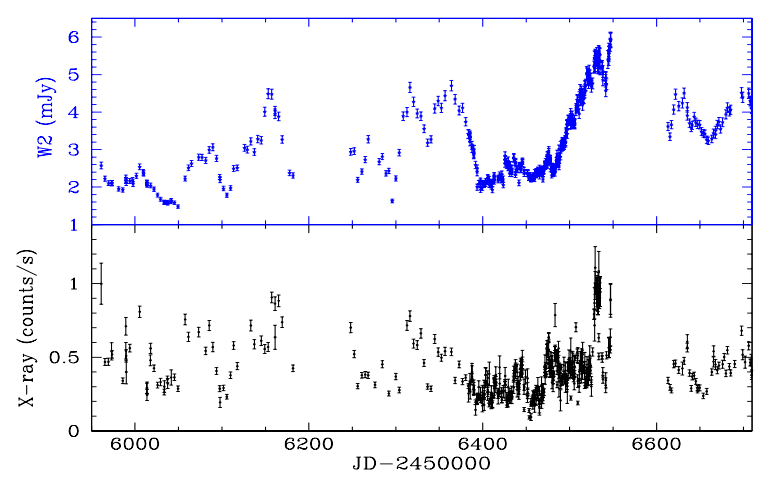

Fig. 6: Long timescale Swift X-ray (lower panel) and UVW2 (upper panel) observations of NGC5548 (McHardy et al. 2014).

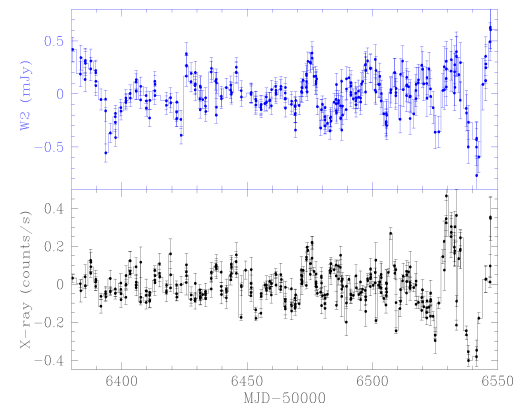

Fig. 7: Swift X-ray (lower panel) and UVW2 (upper panel) for Fig. 8: Lag of a $\sim 150 \mathrm{~d}$ period of twice-daily X-rays by UVW2 observations of NGC5548 in NGC5548 ( $\mathrm{M}^{\mathrm{c} H a r d y}$ et al. 2014) with a from the data 20d running mean subtracted from shown in Fig.7. both.

$\mathrm{M}^{\mathrm{c} H a r d y}$ et al. (2014) presented the result of almost 3 years of Swift monitoring of NGC5548 with $\sim 10 \times$ more observations than those of Shappee et al. In order to follow the Swift preference to use the 'filter of the day', most of the UVOT observations were in the UVW2 band (Fig. 6) which shows a strong ( $>99.99 \%$ confidence) correlation with the $\mathrm{X}$-rays. There are, however, trends in the UVW2 emission, lasting for a few months, which are not present in the X-ray emission. Such trends may arise from intrinsic disc variations caused by inwardly propagating accretion rate fluctuations. Such long term trends, which are not present in both lightcurves, can distort cross correlation functions and so, to measure lags on shorter timescales, it is recommended practice to remove such trends by subtracting a running mean (Welsh 1999). From a 150d period containing 300 observations $\mathrm{M}^{\mathrm{c}}$ Hardy et al. therefore subtracted a $20 \mathrm{~d}$ running 
mean from both lightcurves and a strong X-ray/UVW2 correlation is then seen (Fig. 7).

The lag between the two bands was measured using a variety of techniques (e.g. ZDCF, Alexander 2013), all showing that the UVW2 lags behind the X-rays by about $1 \mathrm{~d}$. In Fig. 8 we show the lag $\left(0.70_{-0.27}^{+0.24} \mathrm{~d}\right)$ as measured using Javelin ( $\mathrm{Zu}$ et al. 2011). These observations provided the first unambiguous evidence that the UV variability was both strongly correlated with the $\mathrm{X}$-ray variations and lagged behind the X-ray variations.

Javelin was designed to improve continuum-line lag measurements in AGN. It assumes that the line lightcurve is a scaled, smoothed and displaced version of the continuum. It models the variability as a damped random walk (DRW), to interpolate between gaps, and directly compares simulated line lightcurves with the observed line lightcurve to recover the lag. Pancoast et al. (2014) show that Javelin recovers simulated lags very well. It might be argued that a DRW, which has a long timescale power spectral slope, $\alpha_{L}$, of 0 , does not describe the long timescale (months/years) X-ray variability of AGN $\left(\alpha_{L}=-1\right.$, e.g. $\mathrm{M}^{\mathrm{c} H a r d y}$ et al. 2005, 2004) very well. However on short timescales the DRW and X-ray power spectral slopes are similar so there is no obvious reason why Javelin should be less applicable here than in the measurement of continuum-line lags.

$\mathrm{M}^{\mathrm{C}}$ Hardy et al. (2014) also present observations of NGC5548 in the 5 other UVOT bands thereby allowing the best measurement at that time of lag as a function of wavelength (Fig. 9). In this case the fit goes straight through the $\mathrm{X}$-ray point with no offset, with $\beta=1.23 \pm 0.31$, in very good agreement with a reprocessing model.

\subsection{Comparison of Lags with Models}

In Fig. 9 we also show the expected model lags following impulse X-ray illumination of a standard thin SS disc for the accepted black hole mass, accretion rate and illuminating X-ray luminosity of NGC5548. Here the model lags are defined by the time after the initial X-ray impulse illumination for half of the reprocessed light to be received. The observed lags are factors of $\sim 3 \times$ larger than the model lags. Only by invoking a much hotter accretion disc, eg by assuming a much higher accretion rate or illuminating $\mathrm{X}$ ray flux than currently accepted values, can we push the standard model close to agreement with the observations. Later observations of NGC5548 by Edelson et al. (2015) and Fausnaugh et al. (2015) with extended UV and optical monitoring confirmed this result and Troyer et al. (2015) find a similar result in NGC6814. However although initially surprising, microlensing observations (Morgan et al. 2010) have also indicated that accretion discs might be factors of a few larger than predicted by SS discs. A possible explanation is an inhomogeneous disc temperature structure. Hotter clumps at large radii can enhance the emission at those radii, making the disc appear larger by factors of a few, depending on the degree of clumpiness (Dexter \& Agol 2011).

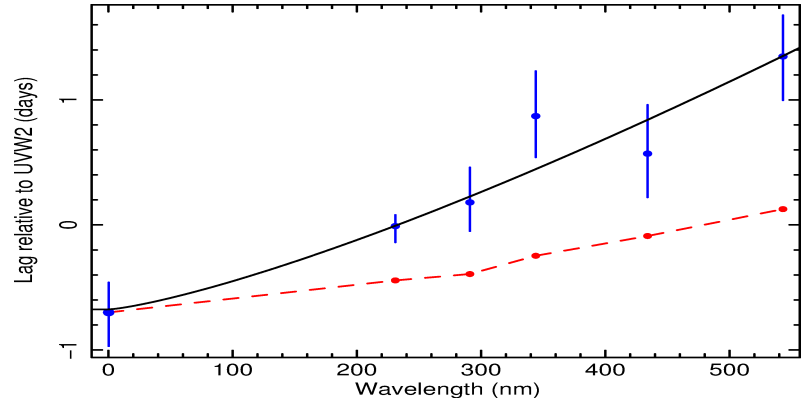

Fig. 9: Lags relative to the UVW2 band for NGC5548 (from $\mathrm{M}^{\mathrm{c}}$ Hardy et al. 2014). The solid line is the best fit through all of the data, including the X-ray point. The fit of $l a g \propto \lambda^{\beta}$ gives $\beta=1.23$, in good agreement with $\mathrm{X}$-ray reprocessing. However the expected lags, assuming a standard thin disc (Shakura \& Sunyaev 1973), are $\sim 3 \times$ shorter (dashed red line) than observed.

\section{XMM-Newton and Ground Based Observations of NGC4395}

As we only have good lag measurements for one AGN, it is very important to make lag measurements on other AGN to determine whether NGC5548 is just unusual or whether standard SS disc theory is incomplete. Lag measurements on larger mass AGN require long observational campaigns. However for smaller mass AGN such as NGC4395, the expected lags can be very well measured in long observations with XMM-Newton using EPIC for X-rays in combination with the OM in fast readout mode for the UV/optical. The fast readout mode has not been widely used for AGN observations but allows continuous readout with sub-second resolution. The suggested 400s UV lag in NGC4395 cannot be detected with standard OM imaging observations with $\sim$ 1100s time resolution. On 28 and 30 December 2014 we therefore observed NGC4395 for $\sim 53 \mathrm{ks}$ each time with XMM-Newton. We observed with the OM in the UVW1 band thus extending our coverage to shorter wavelengths than can be observed from the ground. This band has the highest sensitivity of the UV bands and less host galaxy contamination than the optical bands.

During an XMM-Newton observation a source is typically only observable from the ground for $\sim 4 \mathrm{hr}$. To provide simultaneous g-band observations we therefore made CCD imaging observations with either 100 or 200s integrations depending on telescope size at 6 different ground based observatories (LCOGT McDonald Observatory, Texas; Whipple Observatory, Arizona; LCOGT Haleakala Maui; Kanata Observatory, Japan; ARIES observatory, India and the Wise Observatory, Israel). In Fig. 10 we show the X-ray, UVW1 and combined g-band lightcurves from 30 December 2014. A good correlation is seen between all bands. In Figs. 11 and 12 we show the DCFs between the X-ray band and the UVW1 and g-band lightcurves respectively, confirming high significance correlations. 
To refine the lags relative to the X-rays we calculate lag probability distributions using Javelin for both the UVW1 (Fig. 13) and g-bands (Fig. 14). The resultant lags are $473_{-98}^{+47}$ and $788_{-54}^{+44}$ s. In Fig. 15 we plot both the UVW1 and g-band lags as a function of wavelength. If we force the fit through zero, a simple linear fit (ie $\beta=1$, red line) is best although $\beta=4 / 3$ (blue line) is also an acceptable fit. These observations indicate that reprocessing of X-rays is also responsible for the UV/optical variability of NGC4395.

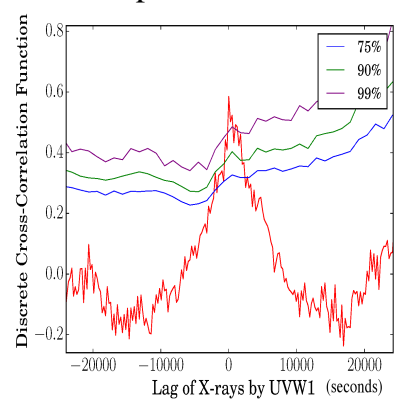

Fig. 11: DCF between the X-ray and UVW1 data for NGC4395 shown in Fig. 10 Simulation based $75 \%, 90 \%$ and $99 \%$ confidence levels are shown.

In Fig. 16 we compare the expected radial emissivity profile of the disc with the distances derived from the lag measurements, following Lira et al. (2011). The distances derived from lag measurements are close to the expected peak emissivity regions. Due to computational problems we have not yet derived lags in the same way as for NGC5548 (Fig. 9) but Fig. 16indicates that, for NGC4395, the agreement between standard SS thin disc theory and model may be closer than for NGC5548.
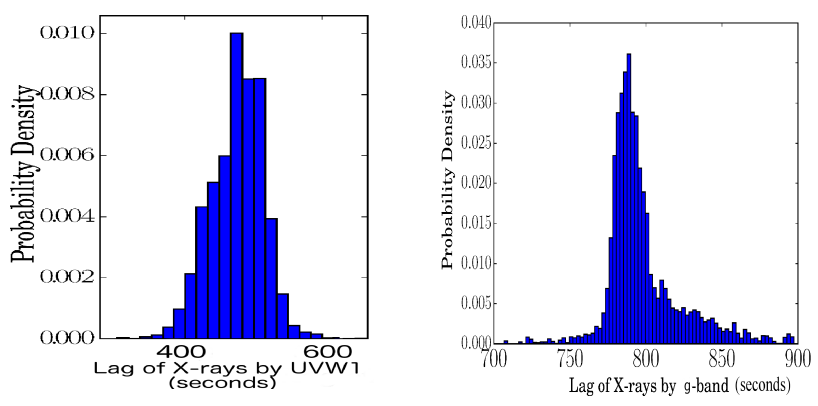

Fig. 13: Lag of X-rays by UVW1 for NGC4395 from the data shown in Fig. 10] using Javelin.

Fig. 14: Lag of X-rays by g-band in NGC4395 from the data shown in Fig. 10 using Javelin.

\section{Conclusions}

To test whether the X-ray to UV/optical lags in an AGN which is quite different to NGC5548 are also larger than

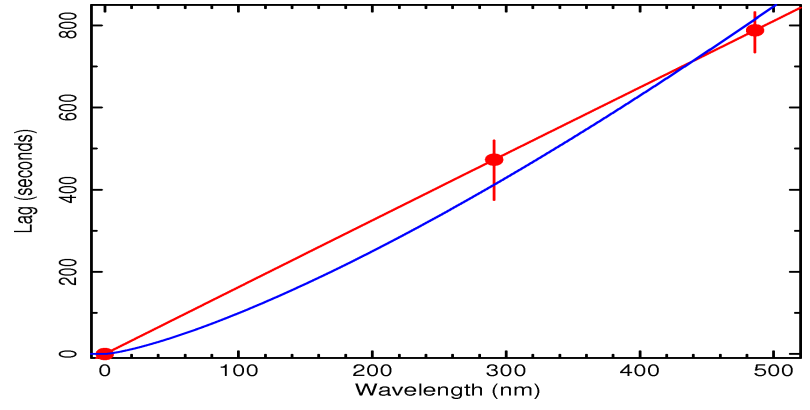

Fig. 15: Lags of UVW1 and g-band behind the X-rays in NGC4395 derived from the data shown in Fig. 10, For $\operatorname{lag} \propto \lambda^{\beta}$, the best fit, if forced through the origin gives $\beta=1.0$ (red line). However $\beta=4 / 3$ (blue line) is also an acceptable fit.

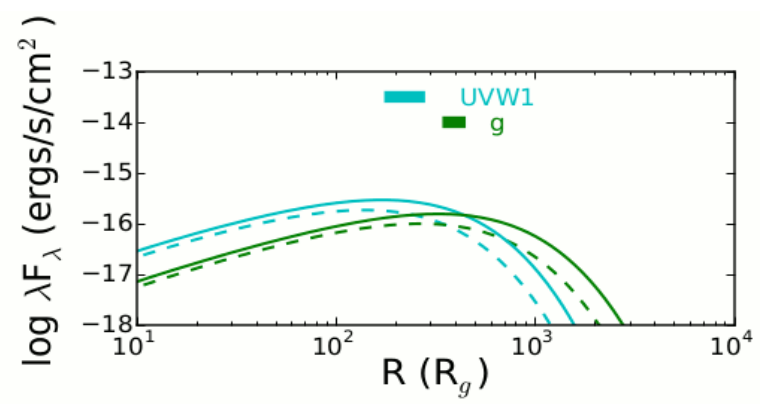

Fig. 16: Emissivity of the accretion disc as a function of radius in NGC4395 for the g-band (dark green) and UVW1 (turquiose) bands (following Lira et al. 2011). We assume $M=3.6 \times 10^{5} M_{\odot}, \dot{m}_{E}=0.0013$ and $R_{i n}=3 R_{g}$. We also assume $L_{2-10}=2.8 \times 10^{42} \mathrm{ergs} \mathrm{cm}^{-2} \mathrm{~s}^{-1}$ and extrapolate from 0.1 to $500 \mathrm{keV}$ but also assume a high albedo so that the power absorbed by the disc is approximately equal to just $L_{2-10} \mathrm{keV}$. The solid lines are the total luminosity released by the disc and the dotted lines are just the gravitational emission.

expected from the standard SS model, we made XMMNewton EPIC and OM observations of NGC4395. This AGN has a mass $\sim 100 \times$ less and accretion rate (in Eddington units) $\sim 20 \times$ less than that of NGC5548. To obtain high enough time resolution in the OM to sample the previously suggested 400s UV lag we used the fast readout mode, not previously used for AGN observations. To obtain the UV lightcurve with the highest possible signal to noise we used the UVW1 filter. To obtain a high quality, continuous, optical lightcurve we chose the g-band and made observations from six different observatories around the globe.

All observations were very successful and we measured lags, relative to the X-rays of $473_{-98}^{+47}$ and $788_{-54}^{+44}$ s for the UVW1 and g-bands respectively. These lags are in good agreement with the hypothesis that the UV/optical variability is driven by reprocessing of X-ray emission. However, unlike in NGC5548, preliminary modelling indicates that the measured lags are not too far different from those ex- 
pected from standard SS disc theory. We remain cautious in our interpretation at present but we do note that the disc in NGC4395 is $~ 50 \%$ hotter than in NGC5548. Increased disc temperature may lead to a more stable disc (e.g. Churazov et al. 2001), less sensitive to radiation-induced perturbations (Pringle 1997).

These observations demonstrate the potential of XMMNewton for X-ray/UV lag measurements in low mass AGN.

Acknowledgements We thank the XMM-Newton operations team for considerable advice and assistance in setting up the observations of NGC4395. We also thank the staff and observers at the McDonald, FLWO, Haleakala, Kanata, Aries and Wise observatories. IMcH thanks STFC for support under grant ST/M001326/1.

\section{References}

Alexander T., 2013, ArXiv e-prints

Arévalo P., Uttley P., 2006, MNRAS, 367, 801

Arévalo P., Uttley P., Kaspi S., Breedt E., Lira P., M Hardy I. M., 2008, MNRAS, 389, 1479

Arévalo P., Uttley P., Lira P., Breedt E., McHardy I. M., Churazov E., 2009, MNRAS, 397, 2004

Breedt E., 2010, PhD thesis, Physics and Astronomy, University of Southampton

Breedt E. et al., 2009, MNRAS, 394, 427

Breedt E. et al., 2010, MNRAS, 403, 605

Cackett E. M., Horne K., Winkler H., 2007, MNRAS, 380, 669

Cameron D. T., McHardy I., Dwelly T., Breedt E., Uttley P., Lira P., Arevalo P., 2012, MNRAS, 422, 902

Churazov E., Gilfanov M., Revnivtsev M., 2001, MNRAS, 321,759

Dexter J., Agol E., 2011, ApJL, 727, L24

Edelson R. et al., 2015, ApJ, 806, 129

Edelson R. A., Krolik J. H., 1988, ApJ, 333, 646

Fausnaugh M. M. et al., 2015, ArXiv e-prints

Lira P., Arévalo P., Uttley P., McHardy I., Breedt E., 2011, MNRAS, 415, 1290

Lira P., Arevalo P., Uttley P., MCHardy I. M. M., Videla L., 2015, ArXiv e-prints

Mason K. O. et al., 2002, ApJL, 580, L117

Morgan C. W., Kochanek C. S., Morgan N. D., Falco E. E., 2010, ApJ, 712, 1129

McHardy I. M. et al., 2014, MNRAS, 444, 1469

M'Hardy I. M., Gunn K. F., Uttley P., Goad M. R., 2005, MNRAS, 359, 1469

M'Hardy I. M., Papadakis I. E., Uttley P., Page M. J., Mason K. O., 2004, MNRAS, 348, 783

Pancoast A., Brewer B. J., Treu T., 2014, ArXiv e-prints

Pringle J. E., 1997, MNRAS, 292, 136

Sergeev S. G., Doroshenko V. T., Golubinskiy Y. V., Merkulova N. I., Sergeeva E. A., 2005, ApJ, 622, 129

Shakura N. I., Sunyaev R. A., 1973, A\&A, 24, 337

Shappee B. J. et al., 2014, ApJ, 788, 48
Smith R., Vaughan S., 2007, MNRAS, 375, 1479

Suganuma M. et al., 2006, ApJ, 639, 46

Troyer J., Starkey D., Cackett E., Bentz M., Goad M., Horne K., Seals J., 2015, ArXiv e-prints

Uttley P., Edelson R., McHardy I. M., Peterson B. M., Markowitz A., 2003, ApJL, 584, L53

Welsh W. F., 1999, PASP, 111, 1347

White R. J., Peterson B. M., 1994, PASP, 106, 879

Zu Y., Kochanek C. S., Peterson B. M., 2011, ApJ, 735, 80 


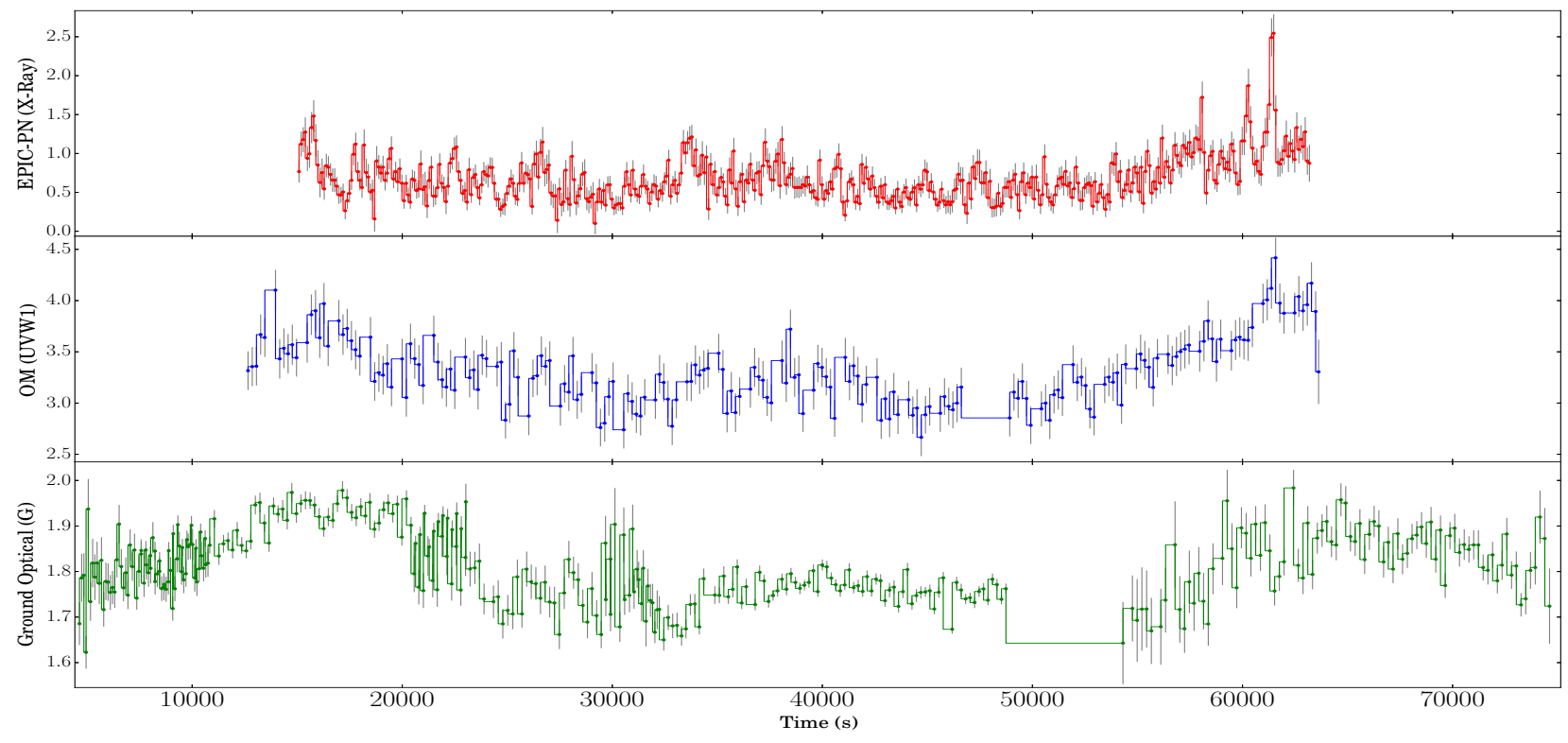

Fig. 10: XMM-Newton EPIC X-rays (top panel), OM UVW1 (middle panel) and ground based g-band (bottom panel) from 30 December 2014 (Connolly et al, in prep). The X-rays are binned to 100s and the UVW1 and g-band to 200s. 